\title{
Teores de sódio e lipídios em refeições almoço consumidas por trabalhadores de uma empresa do município de Suzano, SP
}

\section{Sodium and lipid contents of lunch}

meals consumed by workers of a

company in Suzano, SP, Brazil

Cyntia Kimie Tashira Saldias SALAS ${ }^{1}$

Mônica Glória Neumann SPINELLI ${ }^{1}$

Luciane Mie KAWASHIMA ${ }^{1}$

Aline Miyeko UEDA ${ }^{1}$

\section{R E S U M O}

\section{Objetivo}

Avaliar os valores de sódio e lipídios em refeições almoço consumidas por usuários de uma unidade de alimentação e nutrição.

\section{Métodos}

Análise laboratorial das porções médias consumidas, utilizando-se o método de fotometria de chama para determinação de sódio e o método de extração de lipídios totais por meio de solventes orgânicos. Os resultados foram comparados com as recomendações da Organização Mundial da Saúde e com as novas recomendações do Programa de Alimentação do Trabalhador.

\section{Resultados}

Observaram-se valores de sódio e lipídios acima das recomendações do Programa de Alimentação do Trabalhador. Neste estudo, o valor médio de sódio foi de $2435 \mathrm{mg}$ ( $\mathrm{DP}=518 \mathrm{mg}$ ) e o valor energético médio de lipídios foi de $329 \mathrm{kcal}$ (DP=40kcal) em uma porção média de refeição - 745g (DP=60g).

\section{Conclusão}

São necessárias mudanças para que haja uma adequação das quantidades de sal e de lipídios utilizadas nas refeições, uma redução da utilização de alimentos que contenham grande quantidade de sódio e/ou gordura, e que se programem ações educativas junto aos usuários da unidade de alimentação e nutrição, para a melhoria da qualidade de vida de quem representa a força produtiva da empresa.

Termos de indexação: Consumo de alimentos. Sódio na dieta. Lipídeos. Trabalhadores.

\footnotetext{
1 Universidade de Mogi das Cruzes, Centro de Ciências da Saúde, Curso de Nutrição. Campus I, Av. Dr. Cândido Xavier de Almeida e Souza, 200, 08780-911, Mogi das Cruzes, SP, Brasil. Correspondência para/Correspondence to: M.G.N. SPINELLI. E-mail: <spinelli@usp.br>
} 


\section{A B S T R A C T}

\section{Objective}

The objective of this study was to determine the sodium and lipid contents of lunch meals consumed in a food and nutrition unit.

\section{Methods}

Flame photometry and total lipid extraction with organic solvents were used to determine the amount of sodium and lipids, respectively, per average-size meal. The results were compared with the amounts recommended by the World Health Organization and Brazilian Worker's Food Program.

\section{Results}

Sodium and lipid contents were above those recommended by the Brazilian Worker's Food Program. The mean sodium content was $2435 \mathrm{mg}$ (SD=518mg) and mean energy provided by lipids was $329 \mathrm{kcal}$ (SD=40kcal) per average-size meal (745g, SD=60g).

\section{Conclusion}

Changes are needed to reduce the amounts of sodium and lipids present in these meals, such as using fewer high-fat and/or high-sodium foods. Furthermore, nutrition education should be made available for users of these food and nutrition units in order to improve the quality of life of those who are the driving force of a company.

Indexing terms: Food consumption. Lipids. Sodium, dietary. Workers.

\section{N T R O D U Ç Ã O}

As unidades de alimentação e nutrição (UAN) formam cada vez mais um complexo e importante meio de alimentação de coletividades, apresentando um crescimento significativo nos últimos anos, impulsionado pelo Programa de Alimentação do Trabalhador (PAT), que beneficia oito milhões de trabalhadores em todo o Brasil ${ }^{1-3}$.

Denomina-se UAN o conjunto de áreas com o objetivo de operacionalizar o provimento de refeições, dentro dos padrões dietéticos e higiênicos, às coletividades sadias ${ }^{1}$. Embora os funcionários não sejam obrigados a fazer a refeição no local, esta é a opção natural, pela comodidade, pelo custo e pela qualidade da refeição.

Essas UAN trabalham com distintas formas de distribuição de refeições, dentre as quais o sistema self-service, que permite escolhas de alimentos nem sempre saudáveis ${ }^{1,4}$, uma vez que este nicho de mercado, como qualquer outro, é regulado pela demanda, ou seja, são os consumidores-clientes que, na ponta final do processo, fazem as suas opções ${ }^{5}$. Essa alimentação, muitas vezes, rica em alimentos processados, gorduras e sal, aliada às novas máquinas e equipamentos, que mudam os padrões de trabalho e reduzem o gasto energético dos trabalhadores, têm contribuído com a transição nutricional que vem ocorrendo no Brasil, marcada pelo decréscimo acentuado da prevalência de desnutrição e pelo aumento nas taxas de sobrepeso/obesidade e, conseqüentemente, aumentando o risco nutricional de uma população que tradicionalmente é considerada sadia ${ }^{3,6-12}$. Alguns estudos, como o de Freire \& Salgado ${ }^{13}$ e o de Veloso \& Santana ${ }^{14}$, têm demonstrado que o PAT não vem atingindo o seu objetivo de promoção da saúde por meio da oferta de alimentação saudável, evidenciando uma oferta excessiva de lipídios e energia e elevada prevalência de obesidade e dislipidemias entre os trabalhadores beneficiados pelo programa.

Concomitantemente à transição nutricional, o Brasil está classificado entre os maiores consumidores mundiais de sal, com média de ingestão de 15,09 gramas diários, ou seja, três vezes a mais do limite máximo recomendado pela Organização Mundial da Saúde (OMS)5 ${ }^{5}$ e tem apresentado índices de hipertensão arterial em 18,2\% da população, constituindo a terceira causa da incapacidade temporária dos trabalhadores ${ }^{9,10}$. 
Todas essas condições fazem com que os trabalhadores fiquem expostos aos riscos atribuídos às alterações de consumo decorrentes do padrão alimentar atual. Soma-se a isto o papel do nutricionista dentro das empresas, que é primordialmente administrativo e com ênfase no controle de custos, dificultando possíveis ações de saúde e de educação alimentar na população atendida ${ }^{15}$.

Portanto, com base na preocupação global de aumento da obesidade e da hipertensão, e nas recentes reestruturações sofridas nas recomendações do PAT $^{16}$, este estudo teve como objetivo avaliar os teores de sódio e lipídios totais consumidos pelos clientes de uma UAN, na refeição almoço.

\section{M É T O D O S}

Trata-se de um estudo transversal realizado em uma unidade de alimentação e nutrição localizada no município de Suzano (SP), no período de maio e junho de 2006. Neste período foram escolhidos seis dias, determinados pela possibilidade da pesagem e da normalidade da rotina, em que foram calculadas as porções médias de todos os alimentos consumidos na refeição almoço e a análise laboratorial para determinação da quantidade de sódio e lipídios totais em cada preparação separadamente.

A UAN alvo do estudo foi selecionada por conveniência, visto que este tipo de pesquisa necessita da anuência e do envolvimento da empresa e do nutricionista que administra o serviço de alimentação, uma vez que para a determinação das porções médias consumidas é necessário que se faça a pesagem dos alimentos nas horas de pico da produção, o que interfere no desenvolvimento do serviço.

A UAN é gerenciada por uma empresa terceirizada, que oferece aos seus clientes, na modalidade de auto-atendimento, um cardápio composto por quatro tipos de saladas, dois pratos protéicos (denominados na unidade de pratos principais), uma guarnição, arroz, feijão, sopa, sobremesa (doce e fruta) e opção de ovos ou grelhados (Quadro 1). O cliente pode se servir à vontade, não havendo restrições quanto ao consumo individual. São servidas, aproximadamente, 850 refeições (almoço, jantar e ceia), sendo, em média, 625 almoços, 200 jantares e 25 ceias.

Os funcionários da empresa são, na maioria, homens e adultos, e as atividades na empresa são consideradas leves, pois as tarefas de produção da empresa, de um modo geral, não exigem grande mobilidade e/ou força física.

Durante os seis dias foram avaliadas todas as preparações servidas no almoço. A cada dia, para a quantificação do total de preparação consumida, pesaram-se todas as preparações produzidas no horário do almoço, todas as sobras e os restos, em uma balança com capacidade para $50 \mathrm{~kg}$, sensibilidade $100 \mathrm{~g}$, em que:

Peso total de preparação consumida $=$ preparação produzida - (sobras + restos).

Sobras: alimentos produzidos e não distribuídos;

Restos: alimentos distribuídos e não consumidos ${ }^{1}$

O peso da porção média foi obtido utilizando-se a seguinte fórmula:

Peso total da

Peso da porção média $=\frac{\text { preparação consumida }}{\text { Número de clientes }}$

Depois de calculado o peso médio consumido de cada alimento foi determinado o peso médio total da refeição consumida.

Para a realização da análise laboratorial foram coletadas 100 gramas de cada preparação, que foram pesadas em balança eletrônica, com capacidade para $6 \mathrm{~kg}$, sensibilidade de $2 \mathrm{~g}$ e acondicionadas em sacos plásticos devidamente identificados.

Posteriormente, cada amostra foi triturada em processador de alimentos doméstico e mantida congelada até o momento da análise. 
Quadro 1. Cardápio oferecido em unidade de alimentação e nutrição. Suzano (SP), 2006.

\begin{tabular}{|c|c|c|c|c|c|c|}
\hline Refeições & $11 / 5 / 2006$ & 19/5/2006 & $25 / 5 / 2006$ & $31 / 5 / 2006$ & $12 / 6 / 2006$ & 20/6/2006 \\
\hline Prato protéico 1 & Bife a rolê & $\begin{array}{l}\text { Filé suíno } \\
\text { acebolado }\end{array}$ & $\begin{array}{l}\text { Supremo de frango } \\
\text { especial }\end{array}$ & $\begin{array}{l}\text { Estrogonofe de } \\
\text { carne }\end{array}$ & $\begin{array}{l}\text { Bife de pêra } \\
\text { acebolado }\end{array}$ & $\begin{array}{l}\text { Peixe ao leite de } \\
\text { coco }\end{array}$ \\
\hline Prato protéico 2 & $\begin{array}{l}\text { Lasanha a } \\
\text { bolonhesa }\end{array}$ & Mussaca & $\begin{array}{l}\text { Parafuso com } \\
\text { espinafre ao molho } \\
\text { de creme de leite }\end{array}$ & $\begin{array}{l}\text { Hambúrguer } \\
\text { tropical }\end{array}$ & $\begin{array}{l}\text { Torta de frango } \\
\text { especial }\end{array}$ & $\begin{array}{l}\text { Isca de carne à } \\
\text { oriental }\end{array}$ \\
\hline Guarnição & $\begin{array}{l}\text { Escarola ao } \\
\text { bacon }\end{array}$ & $\begin{array}{l}\text { Couve } \\
\text { refogada }\end{array}$ & $\begin{array}{l}\text { Rolinho de } \\
\text { presunto a } \\
\text { Cristopher Besse }\end{array}$ & Batata palha & Batata frita & Batata corada \\
\hline Prato fixo & Arroz & Arroz & Arroz & Arroz & Arroz & Arroz \\
\hline Prato fixo & Feijão & Feijão & Feijão & Feijão & Feijão & Feijão \\
\hline Salada & Alface & Alface & Alface & Alface & Alface & Alface \\
\hline Salada & $\begin{array}{l}\text { Soja com } \\
\text { espinafre }\end{array}$ & Abobrinha & Escarola & Salada chinesa & $\begin{array}{l}\text { Berinjela } \\
\text { curtida }\end{array}$ & Beterraba \\
\hline Salada & $\begin{array}{l}\text { Maionese } \\
\text { de legumes }\end{array}$ & Agrião & $\begin{array}{l}\text { Tomate com } \\
\text { manjericão }\end{array}$ & $\begin{array}{l}\text { Abóbora } \\
\text { japonesa }\end{array}$ & Tricolor & $\begin{array}{l}\text { Soja ao } \\
\text { vinagrete }\end{array}$ \\
\hline Salada & $\begin{array}{l}\text { Repolho } \\
\text { com abacaxi }\end{array}$ & $\begin{array}{l}\text { Salada } \\
\text { colorida }\end{array}$ & $\begin{array}{l}\text { Maionese de } \\
\text { legumes }\end{array}$ & $\begin{array}{l}\text { Batata com } \\
\text { brócolis }\end{array}$ & Suíça & Chinesa \\
\hline Sopa & Canja & Pavessa & Fubá com couve & Juliana & $\begin{array}{l}\text { Sopa de carne } \\
\text { com legumes }\end{array}$ & Creme suíço \\
\hline Molho & Rosé & $\begin{array}{l}\text { Tomate com } \\
\text { manjericão }\end{array}$ & Rosé & Vinagrete & Vinagrete & Tártaro \\
\hline Sobremesa (doce) & $\begin{array}{l}\text { Maria mole } \\
\text { de maracujá }\end{array}$ & $\begin{array}{l}\text { Sonho com } \\
\text { recheio de } \\
\text { chocolate }\end{array}$ & Mariatina & Maria mole & Curau & Canjica \\
\hline Sobremesa (fruta) & Mamão & Abacaxi & Mamão & Tangerina & Mamão & Melancia \\
\hline
\end{tabular}

As análises laboratoriais foram realizadas na Universidade de Mogi das Cruzes, no período de maio a julho de 2006. Cada alimento foi analisado separadamente.

Para a determinação de sódio foi utilizado o método de fotometria de chama ${ }^{17}$. Pesaram-se $5 \mathrm{~g}$ de amostra homogeneizada em cápsulas de porcelana, que foram calcinadas a seguir em temperatura de 580 a $600^{\circ} \mathrm{C}$ por $10-12$ horas. As cinzas obtidas foram solubilizadas em ácido clorídrico 1:1 (v/v) e transferidas quantitativamente para balões volumétricos apropriados, completando-se o volume com água deionizada. A análise quantitativa das amostras mineralizadas foi feita seguindo a sistemática clássica. Foi utilizada solução estoque de padrão em concentração de 1000,0 $\mathrm{mgL}^{-1}$, preparada a partir de cloreto de sódio. A solução foi preparada com água deionizada imediatamente após a secagem do sal por
24 horas a $110^{\circ} \mathrm{C}$. Esta solução foi então diluída com água deionizada e foi utilizada uma curva analítica entre 0 e $100 \mathrm{mgL}^{-1}$ de sódio.

Para a determinação de lipídios totais foi utilizado o método de Bligh \& Dyer ${ }^{18}$, e todas as amostras foram analisadas em duplicata. Foram utilizados $5 \mathrm{~g}$ da massa homogênea para extração a frio dos lipídios totais, levando-se em conta as proporções entre a água presente na amostra e os solventes de extração: clorofórmio e metanol. O extrato clorofórmico purificado foi filtrado em papel de filtro contendo sulfato de sódio anidro para absorver resíduos de umidade. Em seguida foi transferida uma alíquota de, exatamente, $5 \mathrm{~mL}$ do filtrado para béquer de $50 \mathrm{~mL}$, previamente seco e pesado. Colocou-se em estufa para evaporação do solvente e pesou-se em balança analítica, obtendo-se o conteúdo de lipídios totais, expresso em relação ao peso da amostra úmida. 
Feitas as determinações, calculou-se proporcionalmente o total de sódio e lipídios contidos nas porções médias consumidas.

Para verificar a adequação de cada refeição, por se tratarem de valores de uma única refeição e feita no ambiente de trabalho, foram adotados os parâmetros do Programa de Alimentação do Trabalhador ${ }^{16}$. Cumpre ressaltar que estes estão pautados nas recomendações da World Health Organization ${ }^{4}$, utilizando como referência para a refeição almoço o valor de 30 a $40 \%$ das necessidades diárias.

As quantidades de sódio recomendadas pelo PAT devem se manter entre 720 a 960mg durante a refeição almoço.

O PAT recomenda que $25 \%$ da energia fornecida pela refeição seja proveniente de lipídios, ou seja, o valor energético deste nutriente em uma única refeição deveria variar de 160 a 240kcal, considerando-se um valor energético total para as grandes refeições entre 640 a 960kcal (30 a 40\% da recomendação diária de $2000 \mathrm{kcal}$ ).

Este estudo foi realizado após consentimento da unidade de alimentação e nutrição para o registro das quantidades de ingredientes utilizados na preparação, na pesagem dos ali- mentos preparados e na coleta de amostras dos alimentos para análise laboratorial.

Foi garantido o sigilo em relação ao nome da empresa e à devolução dos resultados encontrados. Foram atendidas todas as normas de segurança alimentar e de segurança do trabalho.

\section{RES ULTA DOS}

A empresa possui um cardápio único que atende aos funcionários administrativos e operacionais fornecendo as seguintes preparações na refeição: arroz, feijão, salada de alface, três saladas elaboradas, dois tipos de pratos à base de carnes (pratos protéicos), guarnição, sopa, sobremesa doce e fruta.

O peso médio de refeição encontrado foi de $745 \mathrm{~g}$ com um desvio-padrão (DP) de 60g, variando de $628 \mathrm{~g}$ a $795 \mathrm{~g}$. Este peso médio correspondeu à soma das porções médias de cada alimento oferecido no dia.

As Figuras 1 e 2 mostram as quantidades de sódio e o valor energético proveniente de lipídios encontrados na porção média diária consumida na UAN pesquisada e o valor recomendado pelo PAT.

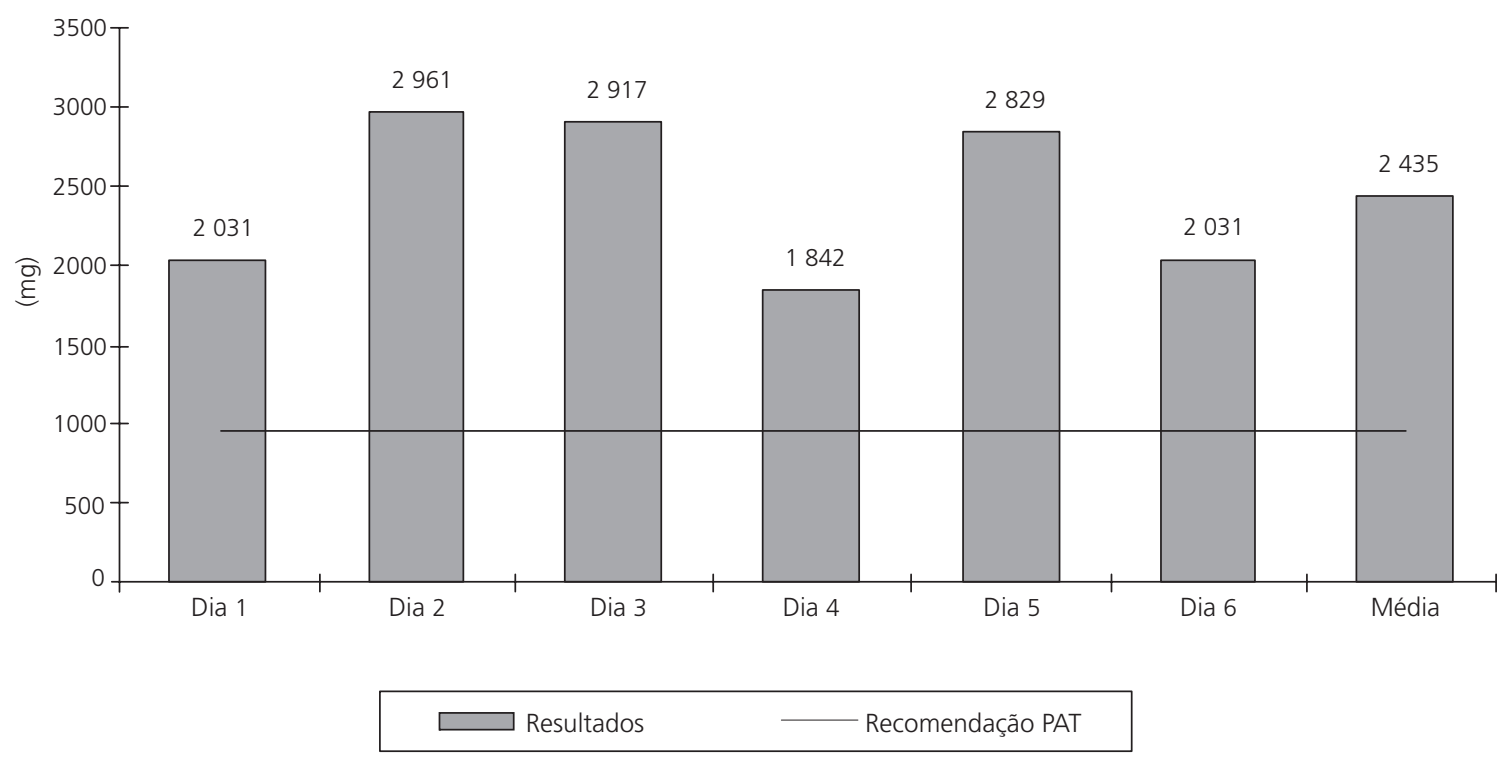

Figura 1. Quantidade de sódio $(\mathrm{mg})$ em peso médio de refeição almoço de unidade de alimentação e nutrição, em relação à recomendação do Programa de Alimentação do Trabalhador. Suzano (SP), 2006. 


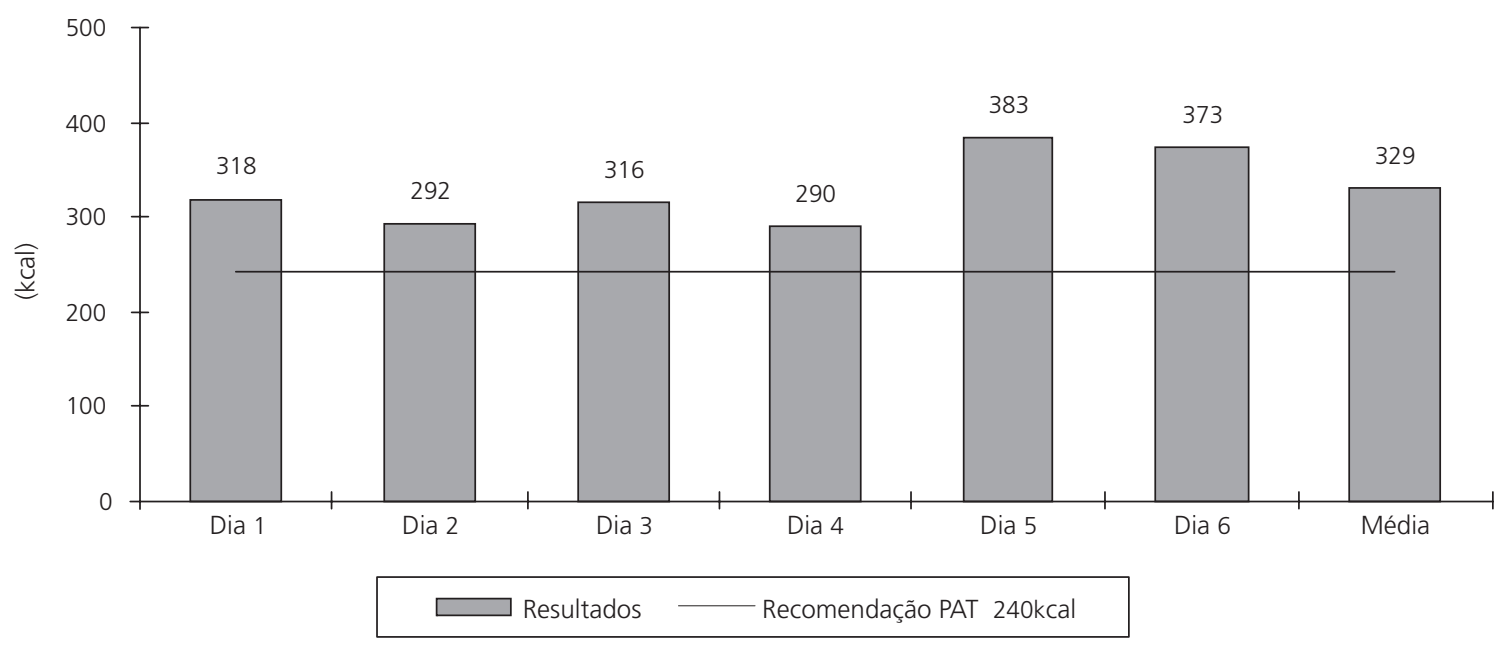

Figura 2. Valor energético de lipídios (kcal) em peso médio da refeição almoço de unidade de alimentação e nutrição, em relação à recomendação do Programa de Alimentação do Trabalhador. Suzano (SP), 2006.

\section{I S C U S S Ã O}

Os estudos de consumo alimentar em UAN são pouco explorados, pois o processo produtivo do preparo das refeições dificulta a coleta de dados, devido ao fluxo intenso de trabalho. Além disso, o pesquisador deve seguir as normas higiênico-sanitárias e as da Comissão Interna de Prevenção de Acidentes (CIPA) durante todo o trabalho.

Para a avaliação do consumo médio de sódio e lipídios totais foi necessário realizar a determinação das porções médias consumidas, porém não foram encontradas na literatura referências que registrem valores mínimos e máximos de consumo por preparações que possibilitem a comparação de resultados.

O presente estudo identificou um consumo médio por pessoa com pouca variação entre os dias avaliados. Segundo Abreu et al. ${ }^{1}$, as pessoas tendem a manter uma certa rotina na alimentação e é possível verificar nas UAN, que têm um público fixo, um consumo bastante uniforme. Salienta-se que a quantidade de sódio e de lipídios é proporcional ao volume consumido, uma vez que a análise dos alimentos foi percentual.

Em relação à média de consumo individual, observa-se que pesquisas realizadas em UAN apresentam valores mais aproximados aos do presente estudo (628g a 795g), como a de Fausto et al. ${ }^{19}$ (619g a 894g) e de Amorim et al. ${ }^{20}$ (609g, $\mathrm{DP}=63,5 \mathrm{~g})$, enquanto em restaurantes comer-ciais por quilo Abreu \& Torres $^{21}$ encontraram uma média menor (454g).

Verifica-se que a refeição, desta unidade pesquisada, apresentou valores elevados de sódio, quando comparados às recomendações do Dietary Guidelines for Americans ${ }^{22}$ e do Ministério da Saúde ${ }^{23}$ de 2 300mg/dia e 2 400mg/dia, respectivamente.

Nota-se, na Figura 1, que os números expressos são baseados no consumo apenas do almoço, por trabalhador, e valores acima de 2 300mg de sódio são encontrados em 4 dias dos 6 analisados, representando, praticamente, o triplo da recomendação do PAT $^{16}$ de $960 \mathrm{mg}$, valor máximo para esta refeição, e quase a totalidade da recomendação diária da Organização Mundial da Saúde ${ }^{4}$. Essa quantidade de sódio é atribuída somente ao sal utilizado para o preparo da refeição e ao sódio dos alimentos. Não foi computado o sódio referente ao sal de adição (distribuído na forma de sachê com $1 \mathrm{~g}$ de sal), que cada usuário poderia utilizar no momento da refeição. 
Spinelli \& Koga ${ }^{24}$ relataram, em um estudo anterior, desenvolvido na mesma unidade, em que não foi avaliado o sódio dos alimentos, um consumo médio individual de sal no almoço de 5,37g, o que corresponde a 2 148mg de sódio, sendo $313 \mathrm{mg}$ provenientes do sal distribuído em sachês. Se acrescentado esse valor de sódio do sal de sachês à média desse nutriente no atual estudo ter-se-á um total de 2 748mg de sódio na refeição almoço.

É preciso observar que a empresa trabalha com um projeto de qualidade de vida e que em uma pesquisa anterior (dados não publicados), nessa mesma unidade, para avaliar a percepção da quantidade de sal na refeição, a maior parte dos usuários se declarou satisfeita com a quantidade de sal utilizada e alguns referiram falta de sal nas preparações.

A hipertensão arterial é observada principalmente em comunidades com alta ingestão de $\mathrm{sal}^{25}$. Essa constatação parece ser independente de outros fatores de risco, como obesidade e alcoolismo. Dessa forma, a restrição de sal na dieta é uma medida recomendada para a população de um modo geral e pode também reduzir em longo prazo, o risco de problemas cardiovasculares $^{26,27}$.

Um inquérito de prevalência de hipertensão feito em 15 capitais brasileiras e no Distrito Federal ${ }^{28}$ encontrou de $7,4 \%$ a $15,0 \%$ de indivíduos hipertensos nas idades entre 25 a 39 anos e $39,0 \%$ a $59,0 \%$ entre 40 e 59 anos, idades semelhantes às da população deste estudo. Considerando que a chance de os indivíduos desenvolverem hipertensão é progressiva com a idade, está claro que existe a necessidade de desenvolver um programa educativo visando à redução do consumo de sódio ${ }^{29}$.

Juntamente com o fator de elevada proporção de sódio na refeição, encontraram-se valores energéticos de lipídios totais maiores do que o máximo preconizado pelo PAT para uma refeição de trabalhador operacional (240kcal), conforme pode ser observado na Figura 2. Por uma limitação do presente estudo não foi possível conhecer o valor energético total da refeição consumida, uma vez que a análise laboratorial se limitou à determinação da quantidade de lipídios totais.

Uma avaliação da porção média oferecida, de cinco dos seis cardápios utilizados nesta pesquisa, por meio de tabelas e de rótulos (nos casos de alimentos não encontrados nas tabelas) ${ }^{30,31}$, mostrou porcentagens de lipídios variando de $42,78 \%$ a $48,06 \%$ do valor energético total (dados não publicados), sugerindo que isto também possa ocorrer na porção média efetivamente consumida.

Outros trabalhos identificam uma alta proporção de lipídios, como o de Abreu \& Torres ${ }^{21}$, nas preparações consumidas em restaurantes comerciais por quilo, o de Fausto et al. ${ }^{19}$, o de Amorim et al. ${ }^{20}$ e o de Höfelmann et al..$^{32} \mathrm{em}$ UAN, e em um restaurante universitário.

Os resultados encontrados corroboram as recomendações de Akutsu et al. ${ }^{33}$ sobre a necessidade da utilização de fichas técnicas de preparo dos alimentos, como forma de controle do valor energético total e dos nutrientes fornecidos e para a promoção da saúde na população atendida.

\section{O N C L U S Ã O}

Conclui-se que, nos dias analisados, em uma única refeição, o valor médio de sódio ultrapassa a recomendação diária e o valor energético médio dos lipídios ultrapassa o valor máximo recomendado pelo Programa de Alimentação do Trabalhador.

São necessárias mudanças no Serviço de Alimentação, com monitoramento constante no processo de produção para que haja uma adequação das quantidades de sal e de lipídios utilizadas para o preparo das refeições e uma redução da utilização de alimentos que apresentem grande quantidade de sódio e/ou lipídios em sua composição.

É preciso que se programem ações educativas junto aos usuários da Unidade de Alimen- 
tação e Nutrição, visando a contribuir com a melhoria da qualidade de vida de quem representa a força produtiva da empresa.

Outros aspectos a serem estudados são as atitudes dos trabalhadores frente à livre escolha dos alimentos e os motivos da resistência à adoção de hábitos alimentares saudáveis.

\section{COLABORADORES}

C.K.T.S. SALAS e A.M.UEDA participaram da coleta de dados, da discussão dos resultados e conclusões e da revisão do artigo. M.G.N. SPINELLI e L.M. KAWASHIMA participaram do delineamento do desenho do estudo, da discussão dos resultados e conclusões e da revisão final do artigo.

\section{REFERÊ NCIAS}

1. Abreu ES, Spinelli MGN, Pinto AMS. Gestão de unidades de alimentação e nutrição: um modo de fazer. 2a. ed. São Paulo: Metha; 2007.

2. Gandra YR, Gambardella AMD. Avaliação de serviços de nutrição. 2a. ed. São Paulo: Sarvier; 1986.

3. Savio KEO, Costa THM, Miazaki E, Schmitz BAS. Avaliação do almoço servido a participantes do programa de alimentação do trabalhador. Rev Saúde Pública. 2005; 39(2):148-55.

4. World Health Organization. Statement on management of hypertension. J Hypertesis. 2003; 21(11):1983-92.

5. Barbosa N. UAN: mercado regido pelo consumidor-cliente [Editorial]. Nutr Prof. 2007; 3(16).

6. Garcia RWD. Práticas e comportamento alimentar no meio urbano: um estudo no centro da cidade de São Paulo. Cad Saúde Pública. 1997; 13(3): 455-67.

7. Giannini SD. Aterosclerose e dislipidemias. Clínica terapêutica: fundamentos práticos. São Paulo: BG Cultural; 1998.

8. Leal MC, Bittencourt SA. Informações nutricionais: o que se tem no país? Cad Saúde Pública. 1997; 13(3):551-5.

9. Oliveira JED, Cunha SFC, Marchini JS. A desnutrição dos pobres e dos ricos: dados sobre a alimentação no Brasil. São Paulo: Sarvier; 1996.

10. Monteiro CA, organizador. Velhos e novos males da saúde no Brasil: a evolução do país e de suas doenças. 2a. ed. São Paulo: Hucitec; 2000.
11. Filho $M B$, Rissin $A$. A transição nutricional no Brasil: tendências regionais e temporais. Cad Saúde Pública. 2003; 19 (Supp 1):S181-S91.

12. Garcia RWD. Reflexos da globalização na cultura alimentar: considerações sobre as mudanças na alimentação urbana. Rev Nutr. 2003; 16(4): 483-92.

13. Freire RBM, Salgado RS. Avaliação de cardápios oferecidos a trabalhadores horistas. Mundo Saúde. 1998; 22(5):298-301.

14. Veloso IS, Santana VS. Impacto nutricional do programa de alimentação do trabalhador no Brasil. Rev Panam Salud Publica. 2002; 11(1):24-31.

15. Ansaloni JA. Situação de trabalho dos nutricionistas em empresas de refeições coletivas de Minas Gerais: trabalho técnico, supervisão ou gerência? Rev Nutr. 1999; 12(3):241-60.

16. Brasil. Ministério do Trabalho e Emprego. Portaria Interministerial $n^{\circ} 66$, de 25 de agosto de 2006. Altera os parâmetros nutricionais do Programa de Alimentação do Trabalhador - PAT. Diário Oficial da União. 2006. 18 ago; (165):153.

17. Association of Official Analytical Chemistry. Official Methods of Analysis of the Association of Official Analytical Chemists. 13th ed. Washington (DC): AOAC; 1980.

18. Bligh EC, Dyer WJ. A rapid method of total lipid: extraction and purification. Can J Biochem Physiol. 1959; 37:911-9.

19. Fausto MA, Ansaloni JA, Silva ME, Garcia Jr J, Dehn AA, César TB. Determinação do perfil dos usuários e da composição química e nutricional da alimentação oferecida no restaurante universitário da Universidade Estadual Paulista, Araraquara, Brasil. Rev Nutr. 2001; 14(3):171-6.

20. Amorim MMA, Junqueira RG, Jokl L. Adequação nutricional do almoço self-service de uma empresa de Santa Luzia, MG. Rev Nutr. 2005; 18(1):145-56.

21. Abreu ES, Torres EAFS. Restaurante "por quilo": vale quanto pesa? Uma avaliação do padrão alimentar em restaurantes em São Paulo, SP. Nutrire: Rev Soc Bras Alim Nutr. 2003; 25:19-34.

22. Department of Health and Human Services. Dietary guidelines for Americans 2005. Available from: $<$ http://www.healthierus.gov/dietary_guidelines>.

23. Brasil. Ministério da Saúde. Secretaria de Atenção à Saúde. Guia alimentar para a população brasileira: promovendo a alimentação saudável. Brasília: Ministério da Saúde; 2005.

24. Spinelli MGN, Koga T. Avaliação do consumo de sal em uma unidade de alimentação e nutrição. Nutrire. 2007; 32(2):15-27. 
25. III Consenso de hipertensão arterial. [acesso 2008 set 21]. Disponível em: <http://www.sbn.org.br/ Diretrizes/cbha7.htm>.

26. Cook NR, Cutler JA, Obarzanek E, Buring JE, Rexrode KM, Kumanyika SK, et al. Long term effects of dietary sodium reduction on cardiovascular disease outcomes: observational follow-up of the trials of hypertension prevention (TOHP). BMJ. 2007; 334(7599):885.

27. Cohen HW, Hailpern SM, Fang J, Alderman MH. Sodium intake and mortality in the NHANES II follow-up study. Am J Med. 2006; 119(3):275.

28. Passos VMA, Assis TD, Barreto SM. Hipertensão arterial no Brasil: estimativa de prevalência a partir de estudos de base populacional. Epidemiol Serv Saúde. 2006; 15(1):35-45.

29. Penner SB, Campbell NR, Chockalingam A, Zarnke $\mathrm{K}$, van Vliet B. Dietary sodium and cardiovascular outcomes: a rational approach. Can J Cardiol. 2007; 23(7):567-72.
30. Tabela Brasileira de Composição de Alimentos. Versão II. 2a. ed. Campinas: Unicamp; 2006.

31. Philippi ST. Tabela de composição de alimentos: suporte para decisão nutricional. 2a. ed. Brasília; 2002.

32. Höfelmann DA, Riekes BH, Azevedo LC. Hábito alimentar e estado nutricional: fatores de risco cardiovascular entre colaboradores internos de uma Unidade de Alimentação e Nutrição. Nutr Pauta. 2005; 70:42-5.

33. Akutsu RC, Botelho RA, Camargo EB, Sávio KEO, Araújo WC. A ficha técnica de preparação como instrumento de qualidade na produção de refeições. Rev Nutr. 2005; 18(2):277-9.

Recebido em: 20/8/2007

Versão final reapresentada em: 23/10/2008 Aprovado em: 30/3/2009 